\title{
Sensitization to 10 mites in a tropic area. Der $p$ and Der $f$ are important risk factor for sensitization to other mites from Pyroglyphidae, Acaridae, Chortoglyphidae, and Glyciphagidae families
}

\author{
Jorge Sánchez, ${ }^{1,2,3}$ Víctor Calvo, ${ }^{1}$ Andrés Sánchez, $, 1,2,3,4$ Susana Díez, ${ }^{1}$ Ricardo Cardona1
}

\begin{abstract}
Background: Much is known about the frequency of sensitization to Blomia tropicalis, Dermatophagoides pteronyssinus and Dermatophagoides farinae, although less is known about sensitization to other species and their possible interactions.

Objective: In patients with allergic manifestations, to evaluate the frequency of sensitization to 10 species of mites in a tropical area and their possible interactions.

Methods: Cross-sectional study. Sensitization was evaluated by skin tests. A generalized linear Poisson regression model with robust variance was used. Based on the sensitization probability reasons and social networking analysis, explorations of relationship for 10 mites were performed. Results: 147 patients were included. The highest sensitization was found to mites' family Pyroglyphidae (> $70 \%$ ) and less frequently was the Glycyphagidae family (<50\%). Sensitization to any mites significantly increased the likelihood of sensitization to others. Sensitization to Der $f$ or Der p increased, more than 20 times the likelihood of sensitization to other mites of the Pyroglyphidae family and more than 10 times to mites from other families. Sensitization to mites from Glycyphagidae, Chortoglyphidae or Acaridae family also increased the risk of sensitization to other mites but less than 5 times.

Conclusion: Sensitization to mites is frequent in tropical area. Pyroglyphidae sensitization is the main risk factor for polysensitization with other mites from Glycyphagidae, Chortoglyphidae or Acaridae. These results must be considered at diagnosis and treatment of allergy diseases.
\end{abstract}

Keywords: Atopy; Allergy; Mites; Allergen; Network analysis; Sensitization

How to cite this article: Sánchez J, Calvo V, Sánchez A, Díez S, Cardona R. Sensitization to 10 mites in the tropic area. Der $p$ and Der $f$ are important risk factor for sensitization to other mites from Pyroglyphidae, Acaridae Chortoglyphidae, and Glyciphagidae families. Rev Alerg Mex. 2017;64(2):153-162
${ }^{1}$ Universidad de Antioquia, IPS Universitaria, Grupo de Investigación de Alergología Clínica y Experimental. Medellín, Colombia.

${ }^{2}$ Fundación para el Desarrollo de las Ciencias Médicas y Biológicas (Fundemed). Cartagena, Colombia.

${ }^{3}$ Universidad de Cartagena, Instituto de Investigaciones Inmunológicas. Cartagena, Colombia.

${ }^{4}$ Facultad de Medicina, Corporación Universitaria Rafael Núñez.

Cartagena, Colombia.

\section{Correspondence: Jorge Sánchez. jotamsc@yahoo.com}

Received: 2016-11-09

Accepted: 2016-12-18 
Abbreviations and acronyms

Aca s, Acarus siro

Blo t, Blomia tropicalis

Cho a, Chortoglyphus arcuatus

Der m, Dermatophagoides microceras

Der $p$, Dermatophagoides pteronyssinus

Eur m, Euroglyphus maynei
GINA, Guidelines for Diagnosis of Asthma

Gly d, Glycyphagus domesticus

GML, generalized linear model

Lep d, Lepidoglyphus destructor

$\mathrm{RP}$, ratio prevalence

Tyr $\mathrm{p}$, Tyrophagus putrescentiae

\section{Background}

Allergies are a group of frequent diseases resulting from interactions between the genetic constitution of everyone and exposure to environmental factors. Although it can occur at any age, onset is usually in childhood and its prevalence appears to be increasing in developing countries, especially those located in the tropics. ${ }^{1,2}$

Atopy is an essential factor for the development of IgE mediated allergy diseases. ${ }^{3,4}$ Currently, the evaluation of atopy is done using extracts from suspicious sources in serological or skin tests measurements. ${ }^{5,6}$ Both procedures usually have good sensitivity and acceptable specificity. In most of the countries located in the tropics, mites are the main cause of atopy but, like pollen grains in Europe, mite species involved vary according to the environmental characteristics of each region, so the epidemiological studies have shown that not all populations are exposed to the same species. ${ }^{7,8}$ In the Latin American tropics much it is known about sensitization to Dermatophagoides pteronyssinus (Der p), Dermatophagoides farinae (Der f) and Blomia tropicalis (Blo t). ${ }^{9,10}$ However, despite that the conditions of this environment favors the growth of a wide variety of species, little has been studied the frequency of sensitization to other mites such as those belonging to the family Acaridae or Chortoglyphidae.

In a study in Egypt, Hossny E et al. evaluated the frequency of sensitization to 5 species of mites in a population of 100 patients diagnosed with asthma between 1 to 7 years, with Der p (11\%) the highest and Acarus siro (4\%) the lowest. ${ }^{11}$ This study found that patients with persistent asthma used to have a sensitization to at least 3 of the mites studied. Puerta et al. ${ }^{12}$ found that among 77 patients with asthma and/or rhinitis positive to Der for Der p, over $70 \%$ had sensitization to other mites (Der f $89 \%$, Ale o $68 \%$ Blo t $80 \%$, Der p $75 \%$ Cho $71 \%$, Lep d
$59 \%$ ), indicating that sensitization in Cartagena is common to Pyroglyphidae mites (Der f, Der p) and mites from other families. Additionally, Puerta L. observed that different IgE binding patterns, suggesting that the sensitization to common allergens with cross-reactivity as allergens species specific occur.

Given the differences in the frequency of sensitization to mites in each environment, we assess the frequency of sensitization and their possible interactions of 10 different mites in a population of patients situated in a Latin American tropical city, enabling better understand of its impact on the diagnosis and treatment of allergies.

\section{Methods}

\section{Geographical and population}

Medellín is a city of Colombia, located at coordinates $6^{\circ} 13^{\prime} 51^{\prime \prime} \mathrm{N}, 75^{\circ} 35^{\prime} 26^{\prime \prime} \mathrm{W}$, between two mountains in the Aburrá Valley, at an altitude of 1479 meters above sea level, with a total area of $380.64 \mathrm{~km}^{2}$ of which 110 are urban ground and 270 rural. Since 2014 its temperature varies in a range between 15$28{ }^{\circ} \mathrm{C}$, with dry seasons during the early and midyear and a relative humidity of $63-73 \%$.

This is a cross-sectional study based on records of a cohort previously formed in Medellín, Colombia. ${ }^{13}$ Briefly, the cohort was formed from 300 patients and for this nested study those subjects who were tasted for the full panel of mites available at the institution and had manifestations of asthma, rhinitis, dermatitis and/or conjunctivitis were selected. Selection was not limited by age or gender. The diagnosis of the diseases studied was carried out following the criteria proposed by various international guidelines such as GINA Guidelines for Diagnosis of Asthma (ginasthma.org), the ARIA guide for diagnosis of rhinitis and/or conjunctivitis and SLAAI guideline for diagnosis dermatitis. ${ }^{14,15}$ 
Evaluation of atopy

The presence of atopy was assessed by skin prick tests using standardized commercial extracts of laboratory Leti (Madrid, Spain) to 10 mites: Blomia tropicalis (Blo t), Der p, Der f, Acarus siro (Aca s), Chortoglyphus arcuatus (Cho), Dermatophagoides microceras (Der m), Euroglyphus maynei (Eur m), Glycyphagus domesticus (Gly d), Lepidoglyphus destructor (Lep d) and Tyrophagus putrescentiae (Tyr p). International recommendations were taken into account for interpretation of skin prick test, considering as positive a wheal greater than $3 \mathrm{~mm}$ relative to the negative control. ${ }^{16}$ All patients before the test discontinued consumption antihistamines or other drugs that might interfere with the result.

\section{Ethical considerations}

The information obtained is strictly adhered to international guidelines from Helsinki protocol, guarding the privacy of patients. Approval by the ethics committee of the institution and the University of Antioquia for review, analysis and dissemination of results was obtained.

\section{Statistical analysis}

Descriptive analyzes were performed for general characteristics of patients through absolute frequencies, relative frequencies and indicators of summaries as the arithmetic mean, standard deviation, quartiles and interquartile range. The criterion of normality of quantitative variables was established by Shapiro Wilk test. By chi square independence test, relationship of demographic, clinical aspects and mites sensitization was established; for the correlation between the sizes of the various mites' wheals the Spearman's rank correlation coefficient was applied.

To control the effect of age, sex and allergic diseases (asthma, rhinitis, dermatitis and conjunctivitis) for sensitization of the mites a generalized linear model (GML) Poisson regression with robust variance was used to adjust the ratio prevalence of sensitization (RP) with their respective $95 \%$ confidence intervals. Based on the probability reasons set, raising from social networking analysis, exploration and linking networks and complex systems of 10 mites was performed. A $p$ value $<0.05$ was considered significant. GEPHI 0.9.1 software, SPSS version 21 and STATA version 13 were used.

\section{Results}

\section{Population characteristics}

147 records were included in this study (Table 1). The average age of patients was 28 years (3-71); $92(61.3 \%)$ were female. In this study, only the records of patients who were tested for 10 mites were included. We note in relation to the historical cohort a difference in terms of mean age ( 20 vs. 28 years), secondary that during skin test in children under 5 years old usually only three mites (Blo t, Der f, Der p) were tested. The most frequently reported disease was rhinitis $(89.8 \%)$, followed by asthma $(50.3 \%)$, conjunctivitis $(48.3 \%)$ and dermatitis $(17 \%)$. $76.8 \%$ of patients had at least two of the diseases studied; only 34 (23.2\%) patients had involvement of a single system (6 single asthma, 25 rhinitis, 1 conjunctivitis and 2 dermatitis). All patients with rhinitis had perennial symptoms.

\section{Frequency of sensitization among mites}

From the 147 patients, 115 were sensitized to mites. Five were mono-sensitized (Blo $\mathrm{t} n=1$, Der $\mathrm{p} \mathrm{n}=$ 2, Der $m n=1$, Lep $d n=1)$. Der f $(76.2 \%)$ and Der p $(73.5 \%)$ were the most frequent. Given the taxonomic classification, we observed a correlation between the frequency of sensitization and the taxonomic closeness of species. Species of the family Pyroglyphidae were the most frequent with an average of $72 \%$ (Table 2), whereas in the family

Table 1. General characteristics of population $(n=147)$

\begin{tabular}{|l|r|r|}
\hline Age (years) & \multicolumn{2}{|c|}{$28 \pm 17$ (3-71) } \\
\hline Female & $\mathrm{n}$ & $\%$ \\
\hline Atopy/mites & $122 / 115$ & $82.9 / 78.2$ \\
\hline Diseases & & \\
\hline Asthma & 74 & 50.3 \\
\hline Rhinitis & 132 & 89.8 \\
\hline Conjunctivitis & 71 & 48.3 \\
\hline Dermatitis & 25 & 17 \\
\hline$>2$ diseases & 114 & 76.8 \\
\hline $\begin{array}{l}\text { Respiratory diseases and } \\
\text { dermatitis }\end{array}$ & 23 & 15.6 \\
\hline
\end{tabular}




\begin{tabular}{|c|c|c|c|c|c|}
\hline Order & \multirow[t]{6}{*}{ Suborder } & Family & Genus & Species & $\%$ \\
\hline \multirow{10}{*}{ Acari } & & \multirow{3}{*}{ Acaridae } & Acarus & Aca s & 61 \\
\hline & & & Tyrophagus & Tyr $p$ & 59 \\
\hline & & & Lepidoglyphus & Lep d & 67 \\
\hline & & \multirow{2}{*}{ Glycyphagidae } & Glycyphagus & Gly d & 49 \\
\hline & & & Blomia & Blo $t$ & 46 \\
\hline & \multirow{5}{*}{ Astigmata } & \multirow{3}{*}{ Pyroglyphidae } & \multirow{3}{*}{ Dermatophagoides } & Der $p$ & 73 \\
\hline & & & & Der $f$ & 76 \\
\hline & & & & Der m & 67 \\
\hline & & \multirow{2}{*}{ Chortoglyphidae } & Euroglyphus & Euro $\mathrm{m}$ & 69 \\
\hline & & & Chortoglyphus & Cho a & 55 \\
\hline
\end{tabular}

Glycyphagidae, only Lep d exceeded $50 \%$ sensitization, unlike Blo t (46\%) and Gly d (49\%) that were below. In the Acaridae family, Aca s (61\%) and Tyr p (59\%) had a similar frequency of sensitization, and in the Chortoglyphidae family, Cho a presented a sensitization of $55 \%$.

We found significant differences in the frequency of sensitization by age group; the ten species of mites had a higher frequency of sensitization in patients $<40$ years old when compared with patients $>$ 40 years old $(\mathrm{p}<0.01$ for each mite). Dividing the groups of patients into quartiles of age $\leq 14$ years (average 7 sensitizations), 15 to 20 years ( 7.8 sen- sitizations), 21 to 39 years ( 6.8 sensitizations), $\geq 40$ years ( 2.9 sensitizations), we observed that patients under age 20 had sensitization to a greater number of mites that patients older than 40 years (Figure 1). There were no differences in the frequency of atopy or number of sensitization to mites by sex.

\section{Wheal size relationship between species}

Wheal sizes reflect the intensity of skin reaction. In assessing whether there was a correlation between the sizes of wheal between different mites, Pyroglyphidae species had a high correlation (Table 3 ), been the highest Der $\mathrm{f}$ and $\operatorname{Der} \mathrm{p}(\mathrm{p}<0.001, \mathrm{r} 0.851)$.

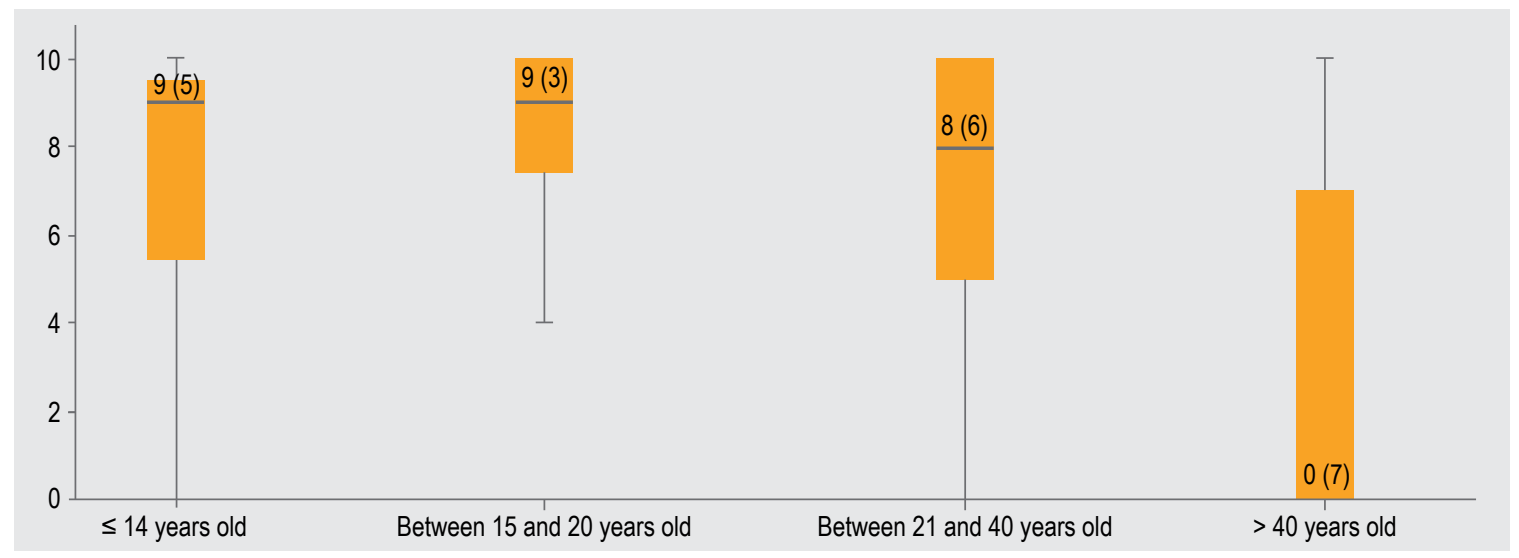

Figure 1. Relationship among age and mites sensitization. Number of mites according to age and mean wheal diameter in parenthesis. 
Table 3. Correlation of the size of the eruption caused by the skin test with mites

Blo t

\begin{tabular}{|c|c|c|c|c|c|c|c|c|c|}
\hline Der $f$ & 0.520 & Der $f$ & & & & & & & \\
\hline Der $p$ & 0.366 & 0.851 & Der $p$ & & & & & & \\
\hline Aca s & 0.458 & 0.725 & 0.668 & Aca s & & & & & \\
\hline Cho a & 0.342 & 0.593 & 0.604 & 0.788 & Cho a & & & & \\
\hline Der $m$ & 0.429 & 0.790 & 0.801 & 0.666 & 0.656 & Der m & & & \\
\hline Eur $m$ & 0.412 & 0.831 & 0.832 & 0.674 & 0.594 & 0.840 & Eur $\mathrm{m}$ & & \\
\hline Gly d & 0.333 & 0.571 & 0.564 & 0.662 & 0.704 & 0.562 & 0.522 & Gly d & \\
\hline Lep d & 0.518 & 0.678 & 0.648 & 0.647 & 0.566 & 0.648 & 0.642 & 0.619 & Lep d \\
\hline Tyr p & 0.404 & 0.666 & 0.634 & 0.703 & 0.680 & 0.663 & 0.615 & 0.656 & 0.671 \\
\hline
\end{tabular}

The family Acaridae showed a high correlation between them ( $p<0.001, \mathrm{r} 0.703)$, but also both species of this family (Tyr $\mathrm{p}$ and Aca $\mathrm{s}$ ) had a close correlation with Cho a from Chortoglyphidae family ( $\mathrm{p}<0.001$, r 0.788 to 0.680 for Aca s and Tyr p). Glycyphagidae species had a moderate correlation between them and low to moderate with other mites. The only exception was Gly d with a high correlation with Cho a $(\mathrm{p}<0.001, \mathrm{r} 0.704)$.

\section{Sensitization reasons among mites}

We found that sensitization to any mites significantly increased the likelihood of sensitization to others (Figures 2 and 3). Sensitization to Der f or Der $p$ increased in general, more than 20 times the likelihood of sensitization to other members of the same family and more than 10 times to mites from other families. Sensitization to Der $m$ or Eur $m$ also increased the risk of sensitization to other mites between 5 to 10 times.

Sensitization to mites from Chortoglyphidae or Acaridae family also increased the risk of sensitization to other mites but less than 5 times. The only exception was the likelihood of sensitization to Cho a an among patients sensitized to Aca s that was 10 times, while the probability of Aca s sensitization among sensitized Cho a was 4 times. In the family Glycyphagidae notable discrepancies were found; Lep d sensitization increased 3 to 8 times the likelihood of sensitization to other mites; while sensitization to Blo $t$ or Gly d increased the probability less than 3 times, including mites of the same family.

\section{Discussion}

Mites found in house dust mostly belong to the suborder Astigmata. Little keratinized cuticle and skin breathing characterize them. The most widely distributed species belong from four families; Acaridae, Glycyphagidae, Pyroglyphidae and Chortoglyphidae. In 1928 Dekker suggested that bronchial asthma associated with house dust could be caused by mites present in the dust. ${ }^{17}$ This hypothesis was confirmed in 1964 by Voorhorst et al., ${ }^{18}$ who demonstrated for the first time that genus Dermatophagoides are responsible of respiratory allergies. Subsequently it is shown that other mites can also influence allergies. ${ }^{19}$

We evaluate in an allergic population frequency of sensitization to 10 mites and their possible interactions. Mite's distribution depends on certain environmental conditions; however, the genus Dermatophagoides has almost cosmopolitan distribution. The family Glyciphagidae by contrast, requires conditions of high temperature and preferably higher humidity, so its distribution is less widespread.

According to what was observed in other studies, we found that mite sensitization more frequently found among patients with allergic manifestations were the 4 species for Pyroglyphidae family. This would indicate that these mites are the most prevalent in the environment of the population evaluated. High co-sensitization of Pyroglyphidae species and high correlation in the size of wheals, suggest that there is also a high rate of cross-reactivity between them. 

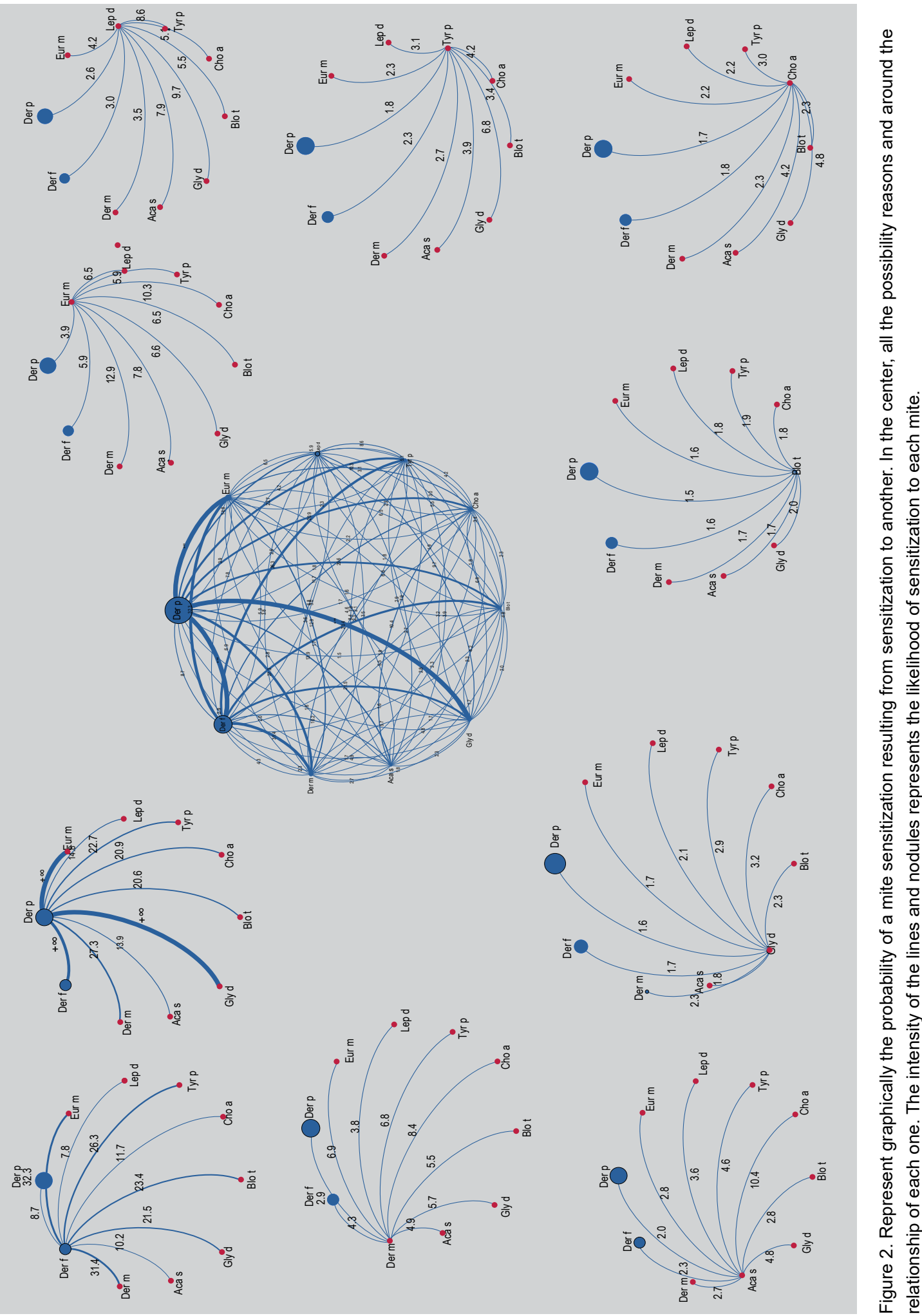


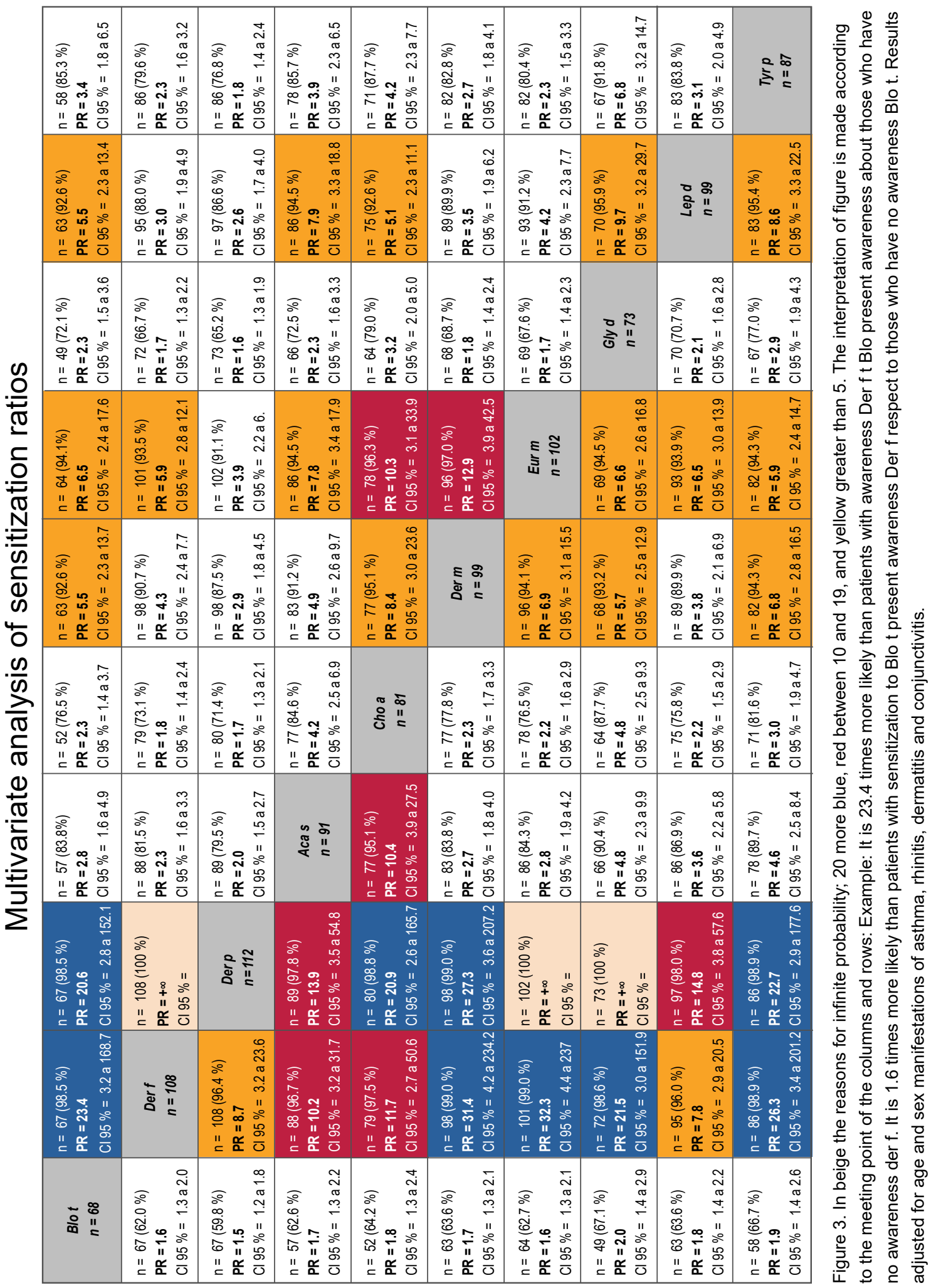


Some studies as Haahtela, et al., ${ }^{20}$ observed a correlation between the size of wheals and clinical relevance; in the cohort PIAMA ${ }^{21}$ the number of sensitizations was directly correlated with patient age, suggesting that early sensitization and intensity of skin reaction are factors that can determine the patient's prognosis. We found that there was a direct relationship between age and the number of sensitizations, up to 40 years, suggesting that early sensitization is an important risk factor for poly-sensitization, perhaps due to chronic inflammatory state, which would facilitate the IgE response to new species especially to allergens with share epitopes with allergens of previous sensitized mites, perpetuating the inflammatory cycle. The lower sensitization in the group over 40 years indicates perhaps a late onset of symptoms, or a loss in the number of sensitizations in time. We believe that the first scenario is more likely because patients over 40 usually referred onset of symptoms after the third decade of life (Data no available). A weakness of the study is not to have a greater number of patients younger than 5 years, which perhaps would identify what was the primary sensitizer mite.

When we assess probability reasons, we found that Der $f$ and Der $p$ were the main risk factor for sensitization to other sources, increasing more than 20 times the likelihood of sensitization to other mites from Pyroglyphidae family and more than 10 times sensitization mites other families. Moreover, this indicates that sensitization to Der $f$ and Der $p$ is a major risk factor for poly-sensitization to other mites and possibly other sources with which it shares proteins, which would explain the high frequency of sensitization to insect and other sources observed in previous studies in the same population. ${ }^{22}$ Additionally we note that sensitization to Der $\mathrm{p}$ confer an increased risk of sensitization to Der $\mathrm{f}$, so between the two, Der p appears to be the main sensitizer. For families Chortoglyphidae and Acaridae the frequency of sensitization was a little lower than for Pyroglyphidae but was present in more than $50 \%$ of the study population.

The correlation of wheal size in the family Acaridae was high (r 0.703), but interestingly both Tyr $\mathrm{p}$ as Aca s had a high correlation with Cho a ( $\mathrm{r} 0.788$ and 0.680 respectively), even higher than that found among them. The taxonomy of mites is complex and is not yet resolved, so the high co-sensitization found between Acaridae and Chortoglyphidae family and the high correlation in the size of wheals, it is may be because taxonomically share common ancestors or principal allergens from both mites are the same. Proteomic alignment studies are necessary to known principal allergens and subsequent verification in vivo to decipher this relationship.

From the mites studied, only Gly d (49\%) and Blo t (46\%) had a sensitization lower of $50 \%$, both from the family Glycyphagidae. Additionally, we observe that the 3 mites of this family had little correlation to the size of the wheal among themselves and with other mites, except for the case of Lep d and Cho a who had a high correlation, which is remarkable because both come from different families. Also, the likelihood of sensitization to other mites among patients sensitized to Gly d and Blo $\mathrm{t}$ was very low compared with mites Pyroglyphidae, Acaridae or Chortoglyphidae family, indicating that principal allergens for these species have low cross-reactivity and therefore specific species.

The above-described results may have important implications for both, the diagnosis and treatment of allergic patients: In the diagnostic battery, is necessary to include species Der $p$ and Der f because they are the most frequently found and are a risk factor for sensitization to other mites; They should also be included Gly d and Blo t because they are sources of sensitization with slight relationship with other mites. For immunotherapy in patients sensitized to multiple mites, Der $\mathrm{p}$ and Der $\mathrm{f}$ should be included in a higher concentration, as they probably represent the initial source of sensitization. With only the administration of this combination specific allergens of Pyroglyphidae family will be included but also the principal allergens with cross-reactivity to other species. However, because Gly d and Blo t appear to represent an independent sensitization, it would be necessary to include in the therapeutic extract if patients are sensitized to them.

In conclusion, the frequency of sensitization to multiple mites is high in the tropics, with sensitization to Der $\mathrm{p}$ as important risk factor for poly-sensitization. It is therefore necessary to identify which sources are present in each region as well as determine which have a real clinical impact. 


\section{References}

1. Chong Neto HJ, Rosário NA, Solé D; Latin American ISAAC Group. Asthma and rhinitis in South America: How different they are from other parts of the world. Allergy Asthma Immunol Res. 2012;4(2):62-67. DOI: http://dx.doi.org/10.4168/aair.2012.4.2.62

2. Solé D, Mallol J, Wandalsen GF, Aguirre V; Latin American ISAAC Phase 3 Study Group.. Prevalence of symptoms of eczema in Latin America: results of the International Study of Asthma and Allergies in Childhood (ISAAC) Phase 3. J Investig Allergol Clin Immunol. 2010;20(4):311-323. Available from: http:// www.jiaci.org/summary/vol20-issue4-num605

3. Kurukulaaratchy RJ, Karmaus W, Arshad SH. Sex and atopy influences on the natural history of rhinitis. Curr Opin Allergy Clin Immunol. 2012;12(1):7-12. DOI: http://dx.doi.org/10.1097/ACI.0b013e32834ecc4e

4. Fasce L, Tosca MA, Baroffio M, Olcese R, Ciprandi G. Atopy in wheezing infants always starts with monosensitization. Allergy Asthma Proc. 2007;28(4):449-453.

5. Bousquet J, Heinzerling L, Bachert C, Papadopoulos NG, Bousquet PJ, Burney PG, et al. Practical guide to skin prick tests in allergy to aeroallergens. Allergy. 2012;67(1):18-24. DOI: http://dx.doi.org/10.1111/ j.1398-9995.2011.02728.x

6. Zakzuk J, Acevedo N, Cifuentes L, Bornacelly A, Sánchez J, Ahumada V, et al. Early life IgE responses in children living in the tropics: A prospective analysis. Pediatr Allergy Immunol. 2013;24(8):788-797. DOI: http://dx.doi.org/10.1111/pai.12161

7. Sánchez J, Diez S, Cardona R. [Frequency of sensitization to animals in a tropical area]. Rev Alerg Mex. 2014;61(2):81-89. Available from: http://revistaalergia.mx/ojs/index.php/ram/article/view/30

8. Burbach G, Heinzerling L, Edenharter G, Bachert C, Bindslev-Jensen C, Bonini S, et al. GA(2)LEN skin test study II: clinical relevance of inhalant allergen sensitizations in Europe. Allergy. 2009;64(10):1507-1515. DOI: http://dx.doi.org/10.1111/j.1398-9995.2009.02089.x

9. Martínez J, Méndez C, Talesnik E, Campos E, Viviani P, Sánchez I. [Skin prick test of immediate hypersensitivity in a selected Chilean pediatric population sample]. Rev Med Chil. 2005;133(2):195-201. DOI: http://dx.doi.org/10.4067/S0034-98872005000200007

10. Caraballo L, Puerta L, Fernández-Caldas E, Lockey RF, Martínez B. Sensitization to mite allergens and acute asthma in a tropical environment. J Investig Allergol Clin Immunol. 1998;8(5):281-284.

11. Hossny E, El-Sayed S, Abdul-Rahman N. Sensitivity to Five types of house dust mite in a group of allergic Egyptian children. Pediatr Allergy Immunol Pulmonol. 2014;27(3):133-137. DOI: Pediatr Allergy Immunol Pulmonol. 2014;27(3):133-137. DOI: http://dx.doi.org/10.1089/ped.2014.0333

12. Puerta L, Fernández-Caldas E, Lockey RF, Caraballo LR. Mite allergy in the tropics: sensitization to six domestic mite species in Cartagena, Colombia. J Investig Allergol Clin Immunol. 1993;3(4):198-204.

13. Sanchez J, Diez S, Cardona R. Sensibilización a aeroalergenos en pacientes alérgicos de Medellín, Colombia Rev Alergia Mex. 2012;59(3):139-147.

14. Brozek JL, Bousquet J, Baena-Cagnani CE, Bonini S, Canonica GW, Casale TB, et al. Allergic rhinitis and its impact on asthma (ARIA) guidelines: 2010 revision. J Allergy Clin Immunol. 2010;126(3):466476. DOI: http://dx.doi.org/10.1016/j.jaci.2010.06.047

15. Sánchez J, Páez B, Macías A, Olmos C, de Falco A. [Atopic dermatitis guideline. Position paper from the Latin American Society of Allergy, Asthma and Immunology]. Rev Alerg Mex. 2014;61(3):178-211. Available from: http://revistaalergia.mx/ojs/index.php/ram/article/view/43

16. Heinzerling LM, Burbach GJ, Edenharter G, Bachert C, Bindslev-Jensen C, Bonini S, et al. GA(2) LEN skin test study I: GA(2)LEN harmonization of skin prick testing: novel sensitization patterns for inhalant allergens in Europe. Allergy. 2009;64(10):1498-1506. DOI: http://dx.doi.org/10.1111 /j.1398-9995.2009.02093.

17. Dekker H. Asthma and mites. J Allergy Clin Immunol. 1971;48(4):251-252.

18. Voorhorst R, van der Hooft-van Asbeck MC, van Krieken H, Nikkels AH. Atopic skin test re-evaluated. V. The wheal-flare ratio of skin reactions to extracts of grass pollen, Dermatophagoides pteronyssinus and to histamine and compound 48/80. Ann Allergy. 1979;42(3):183-184. 
19. Puerta Llerena L, Fernández-Caldas E, Caraballo Gracia LR, Lockey RF. Sensitization to Blomia tropicalis and Lepidoglyphus destructor in Dermatophagoides spp-allergic individuals. J Allergy Clin Immunol. 1991;88(6):943-950.

20. Haahtela T, Burbach GJ, Bachert C, Bindslev-Jensen C, Bonini S, Bousquet J, et al. Clinical relevance is associated with allergen-specific wheal size in skin prick testing. Clin Exp Allergy. 2014;44(3):407-416. DOI: http://dx.doi.org/10.1111/cea.12240

21. Lin W, Gehring U, Oldenwening M, de Jongste JC, Kerkhof M, Postma D, et al. Gas cooking, respiratory and allergic outcomes in the PIAMA birth cohort study. Occup Environ Med. 2013;70(3):187-194. DOI: http://dx.doi.org/10.1136/oemed-2012-101025

22. Rivas A, Kepa J, Gaviria M, Rodrigo. N. Estudio descriptivo de dermatitis de contacto por cosméticos en Medellín, Colombia. Rev Asoc Col Dermatol. 2011;19:262-270. 